\title{
Interstitial pneumonia with autoimmune features: a new classification still on the move
}

\author{
Lorenzo Cavagna ${ }^{1}$, Miguel A. Gonzalez Gay², Yannick Allanore ${ }^{3}$ and \\ Marco Matucci-Cerinic ${ }^{4,5}$
}

\begin{abstract}
Affiliations: ${ }^{1}$ Division of Rheumatology, University and IRCCS Policlinico S. Matteo Foundation, Pavia, Italy. ${ }^{2}$ Rheumatology Division, Hospital Universitario Marqués de Valdecilla, IDIVAL, University of Cantabria, Santander, Spain. ${ }^{3}$ Rheumatology A Dept, Cochin Hospital, Paris Descartes University, INSERM U1016, Paris, France. ${ }^{4}$ Dept of Experimental and Clinical Medicine, University of Florence, Florence, Italy. ${ }^{5}$ Division of Rheumatology, AOUC, Florence, Italy.
\end{abstract}

Correspondence: Lorenzo Cavagna, Division of Rheumatology, University and IRCCS Policlinico S. Matteo Foundation, Viale Golgi, 19, Pavia, PV 27100, Italy. E-mail: Lorenzo.cavagnađunipv.it

@ERSpublications

IPAF classification criteria include several autoimmune conditions with different evolution. The progression into established CTD is common and a continuous up-to-date process of classification criteria of both IPAF and CTD is mandatory. http://ow.ly/pXNW30knUzl

Cite this article as: Cavagna L, Gonzalez Gay MA, Allanore Y, et al. Interstitial pneumonia with autoimmune features: a new classification still on the move. Eur Respir Rev 2018; 27: 180047 [https://doi. org/10.1183/16000617.0047-2018].

In the past decade, the attention has been focused on the relationship between interstitial lung disease (ILD) and connective tissue diseases (CTDs). The clinical impact, as well as the therapeutic approach, classification and diagnosis have been addressed. These two latter items represent an area of great interest because the underlying conditions leading to ILD and the most appropriate treatment still needs to be defined. Recently, the European Respiratory Society/American Thoracic Society proposed the interstitial pneumonia with autoimmune features (IPAF) criteria which represents the effort of pulmonologists to classify patients that may remain clinically undefined [1]. The analysis of these criteria shows that IPAF, similarly to pulmonary arterial hypertension classification [2], may cluster conditions referring to a wide spectrum of rheumatic conditions such as systemic sclerosis, myositis, rheumatoid arthritis and overlap syndromes. These diseases are characterised by highly different characteristics, evolution and therapeutic approaches. Furthermore, IPAF criteria have an intrinsically changing structure, related to the regular update of existing CTD classification criteria and to the even more common identification of new biomarkers in CTDs. By far the most important effect of these criteria is the identification of a grey zone of not well-defined rheumatology conditions, e.g. anti-synthetase syndrome (ASSD) [3]. In this issue of the European Respiratory Review, SAMBATARO et al. [4] address the need to identify specific clinical characteristics and potential areas of improvement in IPAF patients. The starting point of the review is interesting because of the exclusion of papers that included patients diagnosed with ASSD, because no ASSD established classification criteria still exist. The authors' choice is related to the unique characteristics of ASSD, in which it is not unusual to observe the occurrence during follow-up of findings lacking at disease onset. [5]. However, as reported previously [6], in the case of anti-synthetase antibody positivity, the diagnostic scenario may vary with the same patient diagnosed with ASSD if referred to a rheumatologist, or IPAF if referred to a pulmonologist. In fact, a high prevalence of anti-synthetase

Provenance: Commissioned article, peer reviewed.

Received: May 072018 | Accepted: May 302018

Copyright CERS 2018. ERR articles are open access and distributed under the terms of the Creative Commons Attribution Non-Commercial Licence 4.0. 
antibody positivity is reported in the majority of papers evaluated by SAMBATARO et al. [4], thus confirming the fact that despite the presence of this antibody (identifying ASSD) patients may frequently end up being classified as having IPAF. However, some IPAF patients could not be tested for the entire spectrum of antibodies, probably due to the local unavailability of these tests, or because initial anti-Ro antibody positivity might have prompted the clinicians to evaluate further non-anti-Jo-1 myositis specific auto-antibodies. In fact, it is established that anti-Ro antibodies and anti-synthetase antibodies may co-occur in about $50 \%$ of cases, even when patients could be classified as IPAF [7]. Furthermore, we should also consider that if eight anti-synthetase antibodies have been identified to date, at least another 12 aminoacyl-tRNA synthetase enzymes exist, and that the entire spectrum of anti-synthetase antibodies is not covered by any of the existing commercially available tests. All these points clearly indicate the risk that anti-synthetase antibody positivity could be underestimated and that it could be clustered in IPAF. This evidence clearly suggests that well-established and clinically based ASSD classification criteria are needed to reduce the risk of stratifying patients across specialties, in areas that may contribute to real-time incorrectly diagnosed primary disease. Recently, the CLASS (classification criteria of anti-synthetase syndrome) project has been funded by the American College of Rheumatology and the European League against Rheumatism. The aim of the project is to define clinically based criteria of ASSD involving a large number of rheumatology, pulmonology, dermatology, immunology and internal medicine centres expert in the management of rheumatoid arthritis, systemic sclerosis, Sjogren's syndrome and other lung fibrosing conditions. From a scientific point of view, by putting together different specialists we hope to solve the daily discussions about "anti-synthetase antibody positive patient classification" that may delay the correct approach and treatment of affected patients.

SAMBATARO et al. [4] correctly suggest that rheumatologists and pulmonologists should share their experience to optimise the classification of patients affected with CTDs and ILD, as well as all autoimmune conditions potentially underlying an ILD. Furthermore, it is worth noting that IPAF classification criteria include ILD with antibodies such as anti-MDA5 and PM-Scl, but not anti-Ku and anti-Th-To. In this case, it is already widely accepted that anti-MDA5 antibodies are myositis-specific autoantibodies linked with the occurrence of a rapidly progressive ILD, deeply impacting patients' survival (40-55\% after 6 months) [3]. For this reason, the classification of this myositis subset in IPAF is a concern not only because this may be a selection bias in clinical trials but also because it may misguide the therapeutic approach. This area is further complicated by the fact that these patients cannot be classified as polymyositis or dermatomyositis because the new American College of Rheumatology/European League against Rheumatism classification criteria do not include anti-MDA5 antibodies thus pre-empting patients' early classification. Furthermore, the new criteria require more than 6 months of disease duration before their application and they do not include ILD among the identified items [8]. In fact, in anti-MDA5 positive patients, early diagnosis is crucial as we cannot wait 6 months to classify them because they rapidly progress to lung failure. In a recent study the AENEAS (American and European Network of Antisynthetase Syndrome) collaborative group addressed this problem to define the clinical spectrum time-course of MDA-5 positive patients positive. Data collection is ongoing and the hope is to obtain data that will provide information useful to classify patients. Finally, it should be remembered that anti-Ku antibodies are markers of an overlap syndrome, generally with systemic sclerosis [9], whereas anti-Th/To antibodies are commonly identified in systemic sclerosis, even independently to the occurrence of a positive nailfold videocapillaroscopy. Together with some peculiar CTD findings, SAMBATARO et al. [4] correctly suggest these findings as reliable IPAF exclusion criteria. As another example, ANCA-positive patients presenting with an isolated ILD do not satisfy either the existing ANCA-associated vasculitis classification criteria or the IPAF criteria, but this condition surely refers to an autoimmune-mediated ILD. It will be debated as to whether to include these patients within IPAF, even if the clinical picture of these patients is at risk of progression in established ANCA vasculitis. In the future, this group of diseases wait to be more clearly defined by a multidisciplinary approach.

This editorial addresses a point to consider because it is clear that the diagnosis must be achieved very early in these patients to avoid potentially lethal lung disease progression. Moreover, the clash between the need for diagnosis and classification is an evident problem which has prompted pulmonologists to classify the ILD patients that are presently stratified by the rheumatologists in different areas of CTDs as IPAF. It is clear that physicians from the various specialties now need to work together to overcome these problems to improve our understanding about this galaxy of diseases and finally establish shared diagnostic and classification criteria. At present, IPAF criteria have a relevant role in the improvement of our knowledge of ILD in CTDs, and have played a central role in the approval of the CLASS project where the majority of the IPAF team is involved.

In this era, we must be aware that multidisciplinary expertise is necessary, in particular to face patients presenting to the intensive care unit for the first time [10], avoiding the risk of possible confusion with other established rheumatology conditions [11]. In particular, in the early oligo-symptomatic phase of 
systemic sclerosis or myositis when the lung involvement is just starting, an incorrect classification could delay disease modifying treatment which may be deleterious in some highly aggressive subsets [12]. For this reason, this area requires shared expertise as soon as possible to achieve a correct and homogeneous patient diagnosis and classification, thus also reaching agreement on a tailored medical approach to avoid ILD progression.

Conflict of interest: None declared.

\section{References}

1 Fischer A, Antoniou KM, Brown KK, et al. An official European Respiratory Society/American Thoracic Society research statement: interstitial pneumonia with autoimmune features. Eur Respir J 2015; 46: 976-987.

2 Cavagna L, Codullo V, Ghio S, et al. Undiagnosed connective tissue diseases: high prevalence in pulmonary arterial hypertension patients. Medicine (Baltimore) 2016; 95: e4827.

3 Jee AS, Bleasel JF, Adelstein S, et al. A call for uniformity in implementing the IPAF (interstitial pneumonia with autoimmune features) criteria. Eur Respir J 2016; 48: 1811-1813.

4 Sambataro G, Sambataro D, Torrisi SE, et al. State of the art in interstitial pneumonia with autoimmune features: a systematic review on retrospective studies and suggestions for further advances. Eur Respir Rev 2018; 27: 170139.

5 Cavagna L, Nuño L, Scirè CA, et al. Clinical spectrum time course in anti Jo-1 positive antisynthetase syndrome: results from an international retrospective multicenter study. Medicine (Baltimore) 2015; 94: e1144.

6 Monti S, Montecucco C, Cavagna L. Clinical spectrum of anti-Jo-1-associated disease. Curr Opin Rheumatol 2017; 29: 612-617.

7 Scirè CA, Gonzalez-Gay MA, Selva-O'Callaghan A, et al. Clinical spectrum time course of interstitial pneumonia with autoimmune features in patients positive for antisynthetase antibodies. Respir Med 2017; 132: 265-266.

8 Castañeda S, Cavagna L, González-Gay MA, et al. Consideration of antisynthetase syndrome features in classifying patients as having idiopathic inflammatory myopathy: comment on the article by Lundberg et al. Arthritis Rheumatol 2018; 70: 975-976.

9 Rozman B, Cucnik S, Sodin-Semrl S, et al. Prevalence and clinical associations of anti-Ku antibodies in patients with systemic sclerosis: a European EUSTAR-initiated multi-centre case-control study. Ann Rheum Dis 2008; 67: $1282-1286$.

10 Labrador-Horrillo M, Martinez MA, Selva-O'Callaghan A, et al. Anti-MDA5 antibodies in a large Mediterranean population of adults with dermatomyositis. J Immunol Res 2014; 2014: 290797.

11 Cavagna L, Monti S, Grosso V, et al. The multifaceted aspects of interstitial lung disease in rheumatoid arthritis. Biomed Res Int 2013; 2013: 759760.

12 Khanna D, Distler JHV, Sandner P, et al. Emerging strategies for treatment of systemic sclerosis. J Scleroderma Relat Disord 2016; 1: 186-193. 